2020-06

\title{
Removal of babies at birth and the moral distress of midwives
}

\author{
Marsh, W
}

http://hdl.handle.net/10026.1/16037

10.1177/0969733019874503

Nursing Ethics

SAGE Publications

All content in PEARL is protected by copyright law. Author manuscripts are made available in accordance with publisher policies. Please cite only the published version using the details provided on the item record or document. In the absence of an open licence (e.g. Creative Commons), permissions for further reuse of content should be sought from the publisher or author. 


\section{Removing babies at birth and the moral distress of midwives}

\section{INTRODUCTION}

Removing a baby at birth is one of the most challenging aspects of contemporary midwifery practice. Midwives who have to witness, participate and support women through, what has been described as the most draconian action the state can take, describe it as distressing $(1,2,3,4)$. This statutory intervention is an emotive and intrusive event on mothers and their families' lives and one that appears to be increasing in frequency here in the UK $(5,6,7)$.

This paper examines the concept of moral distress, within the context of a doctoral research project that explored midwives' and mothers' perspectives on situations where a baby was removed at birth, or shortly after. The eight midwives who shared their stories as part of this study gave in-depth and emotive accounts of the care they had provided, to women whose babies had been removed at birth. All reported that these experiences had stayed with them far more than any of the more joyful aspects of their work. This paper will focus on the moral distress these midwives experienced, whilst engaging with their work with mothers who had a baby removed at birth.

\section{BACKGROUND}

The term "moral distress" describes the physiological, emotional and psychological suffering that a nurse or midwife experiences, when they are forced to act in ways that are not consistent with their own ethical values and beliefs (8). Moral distress was first identified in 1984 by Jameton (9) in relation to student nurses. He described how the students tended to focus on the "dilemmas of institutional life" (9). 
These dilemmas related to power, relationships, conflict, assertiveness and the consequences of students' proximity to patients and their witnessing patient suffering (10). In an Editorial relating to a special online issue of this journal on moral distress in 2013, McCarthy provided the following definition:

"Moral distress is an umbrella concept that describes the psychological, emotional and physiological suffering that may be experienced when we act in ways that are inconsistent with deeply held ethical values, principles or moral commitments" (8).

In surveying the literature in this journal between 1995 and 2012, McCarthy highlights Corley's "ground breaking research" and Model of Moral Distress (11).

Liashenko's 1995 (12) article was the first to draw attention to moral distress in this journal. One of the earliest studies exploring moral distress in nurses was undertaken in the USA between 1987/1988 by Judith Wilkinson (13). This mixed method study, explored nurses experiences of encountering moral distress and the associated impact of it on themselves and the care of patients. Wilkinson interviewed 24 nurses, identifying clinical situations that had led to their moral distress, for example; providing futile treatment to patients, lying to patients, being socialised to follow orders, fear of losing their job and feelings of self doubt. Nurses in Wilkinson's study also reported the negative feelings they experienced when unable to carry out the behaviour associated with the moral decision they had made (13). The findings of this and other international, qualitative studies $(14,15,16,17)$ support these findings.

Attention is also drawn to theoretical work by Lutzen (18), by McCarthy and Deady (19) and by Repenshek (20). Publications by researchers such Fry (21), Varcoe et al (22), Ohnishi et al (23) and Ping et al (24) have highlighted that moral distress is an 
international phenomenon with significant implications for the wellbeing of the care workforce and the quality of care.

Moral distress has also been measured quantitatively $(14,15)$. Corley $(11)$ developed a moral distress scale to measure the intensity and frequency of moral distress, in nurses working in an intensive care unit (ICU). Questions asked focused on the moral issues that can arise within this clinical environment, that is, carrying out treatment that is deemed futile, understaffing, working in perceived unsafe working conditions and discharging of patients too early. Findings from this study reported that nurses in this environment experienced frequent moral distress and resulted in a negative impact on their lives and emotional wellbeing. It was also reported to have an impact on the quality and safety of patients in their care and a contributing factor in some nurses leaving the profession. The research of Hamric (17), in particular following on from Corley, has been very influential in developing moral distress measurement tools to measure its frequency and the impact it has on nurses, midwives and other professional groups (17). Previous research relating to midwives and moral distress suggests that issues of power and authority contribute to the moral distress of midwives (25).

There have been studies into the role of being a social worker and the moral distress they encounter when working with child protection $(26,27)$. Both the Gray (26) and Leeson (27) studies found that social workers also struggled with engaging with many aspects of child protection work and that the more experienced they were, the better their ability to cope with the distress they feel. This robustness is seen as a positive attribute to have when working with vulnerable families and in a safeguarding and child protection arena (26). However, social workers in this study failed to prioritise 
and explore their emotions, choosing instead to just get on with it, instead of seeking out appropriate support to help them and alleviate the potential for moral distress (26).

Given the impact that moral distress has on professionals and the association it can have on the quality and safety of patient care. It is important that we continue to raise awareness of this phenomenon and find ways of amelioration for professionals who are experiencing it now and preventative strategies for the future generations.

\section{THE STUDY}

\section{Aims}

The overall aim of this research study was to explore mothers' and midwives' experiences of babies removed at birth in a safeguarding context in order to learn from them and contribute to the evidence base for enhancing practice. The aim of this paper is to report midwives' experiences.

\section{Research Design}

A narrative inquiry approach was selected as it afforded space for the participants to share their experiences in their words, which fore grounded the uniqueness of personal experience and the context in which it occurred (28). The participants were recruited via email calls and third party support networks and data collected by undertaking a series of narrative, one to one interviews with mothers and focus groups with midwives, plus the use of photo-elicitation techniques. This involved all participants being invited, with guidance to take photographs before the second focus group/interview where they were invited to share their narrative linked to the images taken and their ascribed meaning (29). Four mothers and eight midwives were recruited to the study and all of them participated throughout the research process. 
This paper reports solely on the recruitment, participation and the findings from focus groups with the midwives. The mothers' experiences are reported elsewhere.

\section{Participants}

Eight midwives were recruited by purposeful sampling.

\section{Recruitment}

Midwives were recruited following an email call through research and children's workforce mail outs across the South Coast of England. The participant information sheet for this population group was also included within the email. Those who wished to take part in the study were asked to contact the researcher directly for further details. Inclusion/exclusion criteria for this group were as follows: Inclusion criteria:

* All registered midwives who have cared for a woman in any part of the childbirth continuum that had her baby removed at birth

Exclusion criteria:

* None

\section{Data Collection}

Focus groups were selected to collect data from the midwives in this study. Focus groups are a recognised data collection tool for qualitative research methods (30). They are particularly effective for bringing together individuals of a similar population or cultural group to discuss topics of shared experience and facilitate the collection of a variety of opinions and views about their experiences.

Each midwife participated in two separate focus groups one month apart, each focus group had 2-3 participants in each (two groups of three and one of two). Groups were purposefully chosen to be small to allow adequate time to share stories and 
due to the emotive nature of their content. Six focus groups were carried out in total with three of them incorporating the use of photo-elicitation. The first focus group lasted approximately one and a half hours and the questions posed were open ended, designed to gather ideas and opinions that are outside the scope of prepared questions. In particular, areas relating to the provision of care to women who have had their babies removed were explored and how they perceived that experience to be. The second focus group involved the sharing and discussion of the images the midwives had taken and how they represented their experiences of caring for a mother whose baby was removed at birth.

Before each focus group commenced, reiteration of key participant information, including confidentiality and the opportunity to withdraw at any time without consequence, took place.

\section{Data Analysis}

Data analysed in this study included transcripts of audio recordings from six focus groups involving eight midwives, eight face to face interviews with four mothers, observational field notes and 40 photographs. The significance and meaning of the images shared by the midwives in this study lay primarily in the interpretation of what they represented to them about their experience of babies removed at birth. These interpretations and insights, shared within the focus groups/ interviews through the dialogues, formed the narrative for analysis.

\section{Ethical considerations}

The research protocol was submitted to a University Ethics Committee in October 2013. Minimal amendments were requested by the committee and a favourable 
ethical opinion was gained from the University Ethics Committee on the $24^{\text {th }}$ of January 2014.

\section{FINDINGS}

The midwives' story: it's more than taking babies, presents the experiences of eight midwives who provided midwifery care to mothers who had their baby removed at birth. The braided stories of these eight midwives are represented in a composite story of five parts; The handover, Providing care in labour, Providing care in the postnatal period, From baby catcher to baby snatcher; and Midwives reflections and memories.

The handover signals the beginning of the midwives' stories and the point at which they received the responsibility of care and meet the mother and her family for the first time. Providing care, in labour and in the postnatal period describes the midwifery care midwives gave during this time and reflects upon the challenges they felt they faced in maintaining some of the core values of being a midwife whilst working as part of a multidisciplinary team who remove babies at birth. From baby catcher to baby snatcher presents the emotional impact upon midwives of engaging with this aspect of child protection work and the impact it had on the midwife/mother relationship and the focus of this paper. Finally, Midwives' reflections and memories, document the thoughts and feelings that have stayed with the midwives involved in this study and the strategies they have employed to cope with their involvement in removing babies at birth.

As discussed this paper is focused upon just one of the subthemes identified, entitled "from baby catcher to baby snatcher" and captures the emotional impact of engaging with this contemporary area of midwifery practice. For the midwives in this study making sense of both their own and the mothers emotions caused them considerable professional and moral distress, even when the decision to remove the baby was deemed to be in the baby's best interest. 
This aim of this paper is to raise awareness of the moral aspects of this area of midwifery practice, alongside the moral distress midwives experience, when caring for mothers whose babies are removed at birth.

\section{The midwives story}

The findings of this research suggest that midwives find, the removal of babies at birth, challenging to witness.

"I cared for a woman whose baby was removed at birth by police officers at birth, it was horrifying. I still get flashbacks, It's almost like post-traumatic thing" (Midwife 1)

"At the end of my shift I just went home and cried myself to sleep. I was pouring with sadness for that woman" (Midwife 2)

Furthermore, if midwives participated personally in the removal of the baby from the mother, they found it emotionally distressing.

"Removing a baby from its mother is one of the most stressful things I have ever done in my career. I didn't know what to do or say" (Midwife 2)

"I did try not to get personally involved but my feelings were running wild and I started to panic saying over and over "I can't do this", don't get me wrong I know I have to safeguard babies but it was horrible, I felt like the child snatcher out of Chitty, Chitty, Bang Bang!!!!" (Midwife, 7)

The midwives also reported significant challenges in their desire to facilitate woman centred care in an institution focused environment.

"A woman I was caring for asked if she could be taken down stairs for a cigarette and I said yes but the senior midwife in charge said under no circumstances could I 
go with her because I was putting myself at risk but I couldn't understand what I was at risk of? The woman had just had her baby removed from her and as a midwife; I just wanted to be with her, to support her" (Midwife 4)

Findings in particular and pertinent to this study were the issues around the imbalance of power between mother and midwife and the feelings of failing to work in partnership with mothers when engaging with child protection activity. In particular, if the midwife was expected to keep secrets from the mother.

"I was told by the social worker not to let Layla know that they were planning to remove her baby that morning, I didn't like being involved in that at all. I did not disagree with the reasoning as to why the baby was being removed, just the lying about it to the woman. I told them I would play no part in it but in the end I did, because I had no choice" (Midwife 4)

The stories shared by the midwives, further indicated that the need to act in the best interests of both the mother and her baby left them feeling that they had betrayed the mother/midwife relationship by prioritising the needs of her baby. They found it particularly difficult to have discussions and make plans about the future for mothers and baby with other professionals if the mother was not actively involved, as it was incongruent with professional philosophy and woman centred care principles $(1,2)$. This was despite the fact that the midwife recognised to do so was in the babies' best interest. They perceived this to be keeping secrets from the mothers that they were expected to have a trusting relationship with and something they found difficult to do.

Finally, the midwife participants reported having heard other midwives speak in a derogatory and unprofessional way about mothers they provided care for. 
"The comments during handover were really cutting and nasty about the woman, a picture was painted of her as some chaotic drug user, it was just wrong" (Midwife 5)

Whilst all of the midwives in this study were clear that in their view this was unprofessional behaviour and poor practice and therefore breached their code of conduct, laid out by the NMC (31), only one midwife observed anyone challenge this behaviour in practice.

"I was really relieved when a senior midwife told them that the comments made were judgemental, not acceptable and unprofessional, I have never seen anyone do that before, most midwives are like me, we know it is wrong but we just ignore it" (Midwife 8)

\section{DISCUSSION}

\section{Moral distress - balancing the needs of both mother and baby}

It could be suggested that the feelings described as betrayal by the midwives in this study, stemmed from the need to shift the focus from woman centred care to child centred care, which can pose both ethical and moral conflicts for midwives (32).

Thompson (33) explored the ethical conflicts midwives face when developing relationships with women and found that there were many challenges that spanned the antenatal, intrapartum and postnatal period with one of them being when the unborn baby was assessed to be at significant risk of harm. Using narrative inquiry Thompson (33) interviewed eight mothers and eight midwives and asked both groups to share their experiences of the perceived dilemmas they faced during their relationships together that had led them to experiencing moral distress.

Midwives do recognise that they have a unique role to play in both safeguarding children and child protection activity, as they are one of the only services that bridge 
the gap between adult focused services like substance misuse services and a child focused service like children's social care. In the UK, the unborn baby has no rights in law and therefore cannot legally be prioritised over the care of a mother as they are not separate until birth (34). This can be illustrated by many cases in the UK where a mother has chosen not to follow the advice of health professionals, in her choices of where to give birth (35). An example of this is women's choices of where to give birth. For example, if the woman or her baby has a condition that significantly raises her or her baby's risk of morbidity or mortality and she chooses to births outside of an obstetric unit and against medical advice. It is therefore interesting to note how women in this context were supported, advocated for by midwives and supervisors of midwives in their choices, by midwives who appear not to experience the same moral distress as is evident when an unborn baby is at risk linked to safeguarding (36).

It is the context of identifying when an unborn baby is at risk of suffering significant harm when born and plans made for removal at birth, where the midwives report the inner moral struggle to prioritise the care of the baby, whilst also balancing the needs of the mother. Therefore, it could be suggested that as long as the mother is at the centre of the care process and able to make informed decisions about the care of herself and her unborn baby the midwife does not face a moral dilemma or suffer any moral distress. In the absence of any research to support this comparison we cannot assume this is the case.

\section{Moral distress in Midwifery Practice}

In this research midwives reported having heard derogatory comments about the mothers they cared for. The midwives' code lays out the professional standards that all nurses and midwives registered in the UK must adhere to (37). This code enables 
mothers to be aware of the standards to expect from the midwifery profession and that are considered to be fundamental to nursing and midwifery practice. Pertinent to this study are the standards laid out in 1.1 which states nurses and midwives should "treat people with kindness, respect and compassion" (37). During the sharing of their stories midwives reflected on observing colleagues failing to uphold this standard. Additionally, standard 3.4 states that all nurses and midwives should "act as an advocate for the vulnerable, challenging poor practice, discriminatory attitudes and behaviour relating to their care" (37).

With only one out of eight participant midwives feeling confident to challenge such behaviour it moves the discussion to consider the wider institutional context of speaking out and having the confidence to challenge. Health care professionals lacking the courage to speak out for fear of being bullied was cited in the Francis report (38) as having led to the hiding of poor practice and substandard care, at a Mid Staffordshire NHS Trust Hospital. It was also reported to have resulted in high patient mortality and neglect (38). In another situation, an investigation team looked at a Lourdes Hospital where an unacceptably high rate of hysterectomies in the postpartum period occurred over a twenty five year period. They concluded that "they found it difficult to understand why so few had the courage, insight or integrity to speak out and say this is not right" (39). The reasons given by the midwives in this study for not speaking out were that it was easier to ignore poor behaviour, as well as the fear of not wanting to offend or upset their co-workers by challenging them, as they still had to have an ongoing working relationship. Moral distress can result in midwives experiencing personal feelings of helplessness, sadness and professional grief that, if left to continue, can result in them leaving the profession (40). Corley (41 found that the moral climate in which nurses work is an important feature in the level 
of moral distress they will suffer and if there is conflict in the ethical values and believes of the individual in comparison to others in the workplace, then the consequences of moral distress will be greater and increase the risk of a nurse or midwife leaving the profession. Cummings (42) supports this and found a correlation between high levels of moral distress in the workplace and nurses leaving the profession.

Four of the midwives in this study had recently left their roles as clinical midwives and moved on to other health care related posts. The reasons they chose to leave was not part of this research and so any feelings of moral distress they experienced could have been a contributing factor to them leaving but cannot be assumed. Moral distress can also affect the quality of care provided by midwives and the avoidance of certain patient groups (42). This was not evidenced in the findings of this study as none of the midwives avoided caring for mothers whose babies were removed. What they did report was that it momentarily affected the way they first approached the mother when they met because of the audible negative of comments they had heard. This may have been observed by the mothers they cared for who may have perceived that the midwives were prejudiced towards them. Chapman (43) claimed that it is impossible for midwives not to make judgements or be prejudiced. Despite the midwives in this study doing their utmost not to have preconceived ideas about the mothers they worked with which all openly admitted, that at some point they had.

\section{Midwife or midspy?}

Safeguarding children and all aspects of child protection are acknowledged as being Everyone's Responsibility (34). During a woman's pregnancy it is the responsibility of the midwife and other professionals involved in her care to identify and act regarding any need for protection of the unborn baby and in relation to the parents capacity to 
"ensure the safety and wellbeing of the baby" (44). Evidence suggests that a multiagency approach is one of the best ways to risk assess and plan the care of an unborn baby who is at risk of significant harm (34). When a mother whose previous history or current social situation deems her as not suitable to provide safe care or a safe environment for her unborn baby, her maternity care will involve a multidisciplinary approach and she will experience being observed and monitored. In this context the midwife will be expected to report concerns and share relevant observations and information with other professionals and agencies as part of her role.

Findings from this research notes how sometimes the midwives were asked not to tell the mother that they were caring for, that they are sharing information with the multi-disciplinary team. They also reporting feeling pressured to collude with other professionals and keeping secrets about the plan for a baby to be removed from its mother at birth. Not informing a mother of the impending removal of her baby resulted in moral distress for all of the midwives in this study. The midwives did recognise the importance of prioritising the safety and wellbeing of the baby but it equally caused the midwives in this study to feel distressed and that they were not being open and transparent with mothers. Furthermore, this emotional and moral conflict challenges the very essence of the mother/midwife relationship. It arguably ruptures the fundamental principles associated with working in partnership with mothers and breaches the very foundations of midwifery practice which is to promote, develop and sustain relationships with women and families based on honesty, trust and open communication $(45,46)$.

Findings in this study support those of Everitt (2), who found that midwives do not always comply with the request to withhold things from the mother, preferring instead 
to use their professional judgement to assess each situation on an individual level and how this might impact upon their relationship with the mother in their care. Midwives used guidance to support their decisions as laid out in their standards for midwifery practice and their code of conduct $(37,47)$. These professional standards operate within the wider context of the law and legislation in relation to maintaining human rights and ensuring that midwives and other professionals make professional, ethical decisions about women in their care whilst maintaining confidentiality and supporting the most vulnerable in our society (48).

Multidisciplinary involvement in care planning can increase potential for conflict and has been cited in the literature as being a significant issue for professionals, working in safeguarding and child protection situations $(49,50)$. Being an advocate for woman in the decision making process was important to the midwives in this study, it caused them distress if they felt that they had been manipulated or used to get close to the mothers on another agency's behalf. For example there was evidence in the findings of this study that several midwives felt that their opinions and assessments of a mother's ability to parent were not taken into account during court proceedings despite being asked to make extensive records of their observations whilst the mother and baby were in their care.

Midwives in this study also reported how their accessibility and mother/midwife or professional friend relationship had been used to collect information. The use of this sometimes limited yet significant observation which would arguably be difficult for any other professional group to gain, as it is enabled by the midwife/mother relationship left midwives angry and despondent that they had let the woman down. These findings have similarities with Wood (1), who also found that midwives appreciate participation in the care planning process and ensuring that the care plan 
represented the needs of women in their care. They had become despondent and angry if they had observed a mother in their care to have jumped through hoops to comply with children's social care and yet they removed her baby from her anyway.

\section{In whose best interest?}

All of the midwives in this study hoped that when a baby was removed at birth from its mother that it must be the right thing to do and thinking that enabled them to cope with their involvement. There is a dearth of follow up data exploring the long term outcomes of babies removed at birth and their mother's lives post removal. $10 \%$ of babies removed from mothers at birth will be returned following court proceedings (5), which raises the question of the quality, process and timing regarding removals of babies from mothers at birth. One of the mothers in this study had her baby returned to her following proceedings and she feels that her baby has suffered attachment issues as a result of their forced separation at birth. This could have been prevented had they been monitored and supported in a mother and baby placement. The dilemma here is the availability and access to such a unit when needed alongside financial sustainability.

The ethical implications of undertaking research regarding removal versus non removal of babies at birth are numerous but some studies in this area have been undertaken. A randomised controlled trial (RCT) of 5000 families was carried out in Minnesota by Loman and Siegel (51) with families being randomly allocated to either the standard social care process or an alternative and supportive approach. The standard approach involved traditional assessments and close monitoring to identify and act upon perceived ineffective or poor parenting. The supportive approach included support and interventions to families to help them achieve what they feel 
they need to parent their baby more effectively. The study continued for three and a half years and findings were, that the supportive approach had not compromised the safety of the children and further evidence to suggest that it had enhanced it. Furthermore, both social workers and families preferred this way of working together and whilst initial costs to set the programme up had been high, it had still proved more cost effective than the costs of removing babies into care and funding court proceedings against their parents. Children who had grown up in the standard social care process experienced more illness than before social workers had become involved, missed more days off school and were more difficult to parent.

The removing of babies at birth or later as younger children has no evidence of benefit (51), suggesting that the midwives in this study cannot be certain that their actions were in the best interests of the baby. Furthermore, there is potential for them to be participating in the causing of comparable harm to both mothers and their babies by separating them before assessments have been completed (51).

\section{CONCLUSION}

The findings of this research fills a much needed gap in the evidence base as to how UK midwives participate in the process of removing babies at birth and the consequence to them of doing so. Midwives who provide care to mothers whose babies are removed at birth experience high levels of moral distress. This is further impacted by the challenges they face in trying to deliver woman centred care in an institution focused environment. It is evident that the more experience and knowledge a midwife had around removing babies at birth, the better her ability is to professionally make sense of it and demonstrate resilience. All of the midwives said that they at times had felt afraid when engaging with this aspect of their work and that the experiences stayed with them for far longer, than any other distressing 
experiences they had encountered during their career above and beyond that of caring for a woman who has had a stillbirth.

The issues discussed here are worthy of future discussion and investigation in order for us all to develop greater understanding of the impact of this statutory intervention on mothers, babies and midwives and how we are able to balance the risks associated with intervention with the risks of ensuring the baby is protected from harm. 
